\title{
Escritura en bordes difusos. \\ Animitas como un dibujo incompleto
}

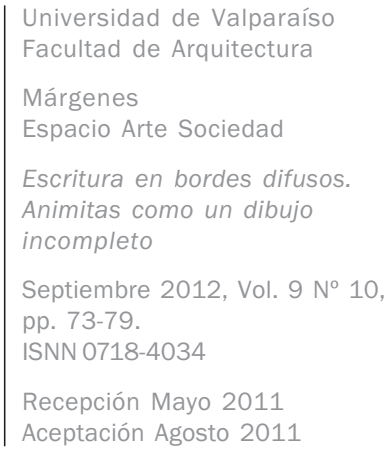

\section{Resumen}

MARÍA PAZ CONTRERAS VALDOVINOS

> Licenciada en Arte Universidad Arcis 2009. Posgrado de Lenguajes Artísticos Combinados, en proceso. Ayudantía para Taller de Borde $\left(5^{\circ}\right.$ semestre) Universidad Arcis

Este texto corresponde a un trabajo de relaciones comparativas entre elementos de religión popular y determinadas manifestaciones en el arte contemporáneo. Estos elementos; la animita por un lado y la instalación y el site specific por el otro, comparten características formales pese a sus distancias en términos de razón y funcionalidad, sin embargo estos elementos compartidos, permiten, desde una producción artística, evidenciarse y convivir en algunas ideas de lo espacial y ritual bajo algunas articulaciones desarrolladas por ejemplo, por Nicolas Bourriaud en su texto Estética Relacional.

La investigación se centra en los elementos que comparten ambas manifestaciones y tiene como resultado una dibujo - instalación en una muestra colectiva en el Centro de Extensión en Identidad y Patrimonio del Consejo Nacional de la Cultura y las Artes en Valparaíso, muestra que se encontraba en el marco del II Encuentro Nacional Animitas, Identidad y Patrimonio.

Palabras claves

frontera permeable, animitas, dibujo, instalación, site specific, estética relacional.

Blurry Writing: Animitas as an incomplete drawing

\section{Abstract}

This text makes a comparison between some religious popular elements and some contemporary artistic work. These elements, on the one hand, animitas, and the installation and site specific, on the other hand, share certain formal characteristics in spite of their differences in terms of motives and functionality. However, these shared elements can be shown and make them coexist in some spaces as well as some rituals. One example of these are some articulations developed by Nicolas Bourriaud in his text Relational Aesthtetics.

This research focused on the common elements of these artistic works. An outcome of this research is a Drawing-installation in a collective exhibition in the Identity and heritage Outreach Center of the Culture and Arts National Council in Valparaíso, in the II National Meeting: Animitas: Identity and Heritage.

Key-words

permeable border, animitas, drawing, installation, site specific, relational aesthetics 


\section{Écriture aux bords diffus. Animitas comme un dessin incomplet}

\section{Résumé}

Ce texte correspond à un travail de relations comparatives entre des éléments de la religion populaire et de certaines manifestations dans l'art contemporain. Ces éléments, les animita d'une part, l'installation et le site specific d'un autre, partagent des caractéristiques formelles malgré leurs distances en termes de raison et fonctionnalité, toutefois ces éléments partagées permettent, depuis une production artistique, être manifeste et coexister dans quelques notions spatiales et rituelles sous quelques articulations développées par exemple, par Nicolas Bourriaud dans son texte esthétique relationnel.

La recherche se concentre sur les éléments qui partagent les deux manifestations et qu' a eu comme résultat un dessin-Installation dans une exposition collective dans le Centre d'Extension d' Identité et Patrimoine du Conseil National de la Culture et les Arts à Valparaiso, exposition qui se trouvait dans le cadre du Ile Rencontre Nationale "Animitas, Identité et Patrimoine".

\section{Mots clés}

frontière perméable, animitas, dessin, installation, site specific, esthétique relationnelle

En todos los juegos anida la idea de la muerte. Jim Morrison en Una Plegaria Americana

La animita no es sólo un elemento de religión popular, sino que como construcción abierta la he identificado en algunas de sus características variables y complejidades propias o espaciales, lo que me permite ubicarla paralelamente con otro tipo de manifestaciones distantes; como lo que podrían ser elementos de arte contemporáneo entre aspectos teóricos y prácticos.

La animita me obliga a curiosearla, agacharme, arrodillarme para entrar en su dinámica. No basta con una detención, una pausa, hay un gesto más determinante del cuerpo en esta relación, así la instalación artística me invita a recorrerla a dejar la añeja condición contemplativa y convertirme en practicante.

Pienso en el lugar del emplazamiento, con las características espaciales y constructivas de la animita. Directamente establezco relaciones entre site specific ${ }^{1}$ y la instalación ${ }^{2}$; ambos conceptos son de relaciones espacio-temporal que adquieren gran importancia en la producción de arte contemporáneo, principalmente por las condiciones propias, de ambas manifestaciones en lo que respecta a la experiencia entre espectador y objeto, y donde el foco está puesto en obras del tipo relacionales (Bourriaud, 2006). Entre éstas consideraciones está la importancia de la producción artística con y para el otro distanciándose de la relación del autor y la autonomía de la obra, adquiriendo en esto el valor de la mirada, el aquí y ahora del emplazamiento; el recorrido del objeto por parte del espectador $-\mathrm{y}$ quien lo constituye obra por medio de ésta mirada-. Asimismo, el público adquiere un lugar diferenciado por la necesidad de su presencia ante el objeto, para que se consolide como objeto de arte. El espectador, es entonces en la actualidad un practicante en el campo del arte contemporáneo.

Por otra parte, un altar, o un espacio diferenciado dentro de una casa -o en la calle- muestra este tipo de características, sin embargo la animita tiene además de la demarcación del espacio, la carga histórica del hecho que la erige. Así, no es un espacio que se ha determinado diferenciar de manera arbitraria, si no que está dado por los acontecimientos del lugar.
No considero totalmente el concepto de intervención (artística) para el caso de la animita, dado que la intervención para varios autores requiere la variación o anulación de alguna función del lugar, situación que en la animita no está claramente identificada, es más, aparece más bien como una situación limítrofe entre la transformación de funciones espaciales, en una especie de convivencia entre usos primarios y usos agregados como los de tipo espiritual o religioso, que coexisten amigablemente.

\section{EL CUERPO. TUMBA-ANIMITA}

Haré una pequeña contraposición entre tumba y animita, para dejar claras las diferencias y cuáles son las relaciones del cuerpo en ambas -cuerpo de quien o quienes hayan fallecido y las relaciones espaciales y corporales de apropiación de los espacios, por quienes mantienen una práctica en torno al recuerdo de aquél que ha muerto-.

La tumba es una relación de cuerpo y superficie. El cuerpo es enterrado, se oculta bajo la tierra por razones higiénicas y porque su descomposición quizás sería una de las cosas que más nos costaría ver en un ser querido. El cuerpo y su historia con final se caracteriza por ser única, lo que sobre la superficie se construye es un recordatorio de aquellas particularidades que nos gustaría siguieran presentes en los vivos de quien ha muerto. Las apropiaciones de esa superficie, tienen que ver con la decoración y/o la comodidad para quienes visitan la tumba, como por ejemplo la construcción de una banca o instalar un toldo para el sol. Es necesario reconocer que entre la práctica de las animitas, también se encuentran tumbas de personajes reconocidos o que tuvieron una muerte trágica y que el colectivo, las resignifica como animitas.

Sobre esto último, sería pertinente señalar que la conciencia de la muerte hace al duelo, como señala Regis Debray, con respecto al nacimiento de la imagen como una extensión de la vida a lo que se podría fácilmente ligar con estas prácticas, ya sea las funerarias como la de las animitas, como sistemas o 
mecanismos para la superación de muerte, pero al mismo tiempo como una manera de tener presente y mantener durante un periodo de tiempo, después del fallecimiento, al difunto en la dinámica de los vivos.

La animita por su parte es una relación distinta entre el espacio y el cuerpo de quien fallece, dado que el cuerpo va al cementerio y lo que la religión popular señala es que el alma es lo que queda perdido y errando en búsqueda de su cuerpo faltante. La animita es una relación de superficie e historia, donde la construcción o la apropiación del espacio con la casita, gruta, cruz u otro, tiene que ver con señalar en el territorio ese evento trágico.

\section{CUERPO HACIENDO LUGAR}

La relación de cuerpo y práctica ha sido desarrollada por varios autores que señalan un acercamiento o reconocimiento del cuerpo como una relación fronteriza entre lo que somos como unidad y eso otro como todo lo que no es nuestro propio cuerpo (Merleau-Ponty, 25), de alguna manera como una frontera que permite el ingreso de cierta información del exterior y la modificación por medio del cuerpo de ese espacio diferenciado (Pérez Riobello, 2008).

Investigar la animita, desde distintas perspectivas como es el caso de esta publicación, exige la relación sensible de la experiencia, lo que se desarrolla y complementa con el estudio teórico, religioso y social de las prácticas que los mismos fieles llevan a cabo. Obviamente ello no significa que cada uno realice, por ejemplo, una conversión religiosa, si no que desde mi lugar de artista puedo observar las prácticas de otros y reconocer en terreno cuáles son mis prácticas y cómo asociarlas en dicho terreno. De esta manera puedo hablar con propiedad (con mi propiedad) de un fenómeno con variado tipo de formas en las que es posible observar, sin obviar $u$ vejar su funcionalidad primera.

Las reflexiones con respecto al espacio que abarca la animita pueden ser leídas, como ya señalaba, desde el cuerpo como una orgánica sensible y perceptiva, es por ello que se asume que las relaciones espaciales las reconocemos por medio de nuestra corporalidad y claramente es a través de ella, que este análisis tiene validez.

A través del cuerpo diferenciamos el arriba del abajo, lo que está cerca o lejos, cuando estamos dentro o fuera de algo. Por otra parte, algunas de estas relaciones espaciales han sido cargadas de elementos culturales que nos determinan algunos comportamientos, por ejemplo aprendemos por medio de la experiencia que hay lugares para determinado tipo de actividades y que hay espacios diferenciados, que aunque no cambien su forma poseen una finalidad distinta como podrían ser los espacios públicos y privados. (Merleau-Ponty 27).

Un ejemplo de esto sería lo que ocurre en un espacio diferenciado como el teatro, donde nos mantenemos alejados del escenario porque sabemos que ese espacio tiene una de esas funciones diferenciadas. Sin embargo, más allá de la evidente separación entre espectadores y público, en la cotidianidad podemos encontrar este tipo de diferenciaciones del espacio por actos simples como encuadrar la mirada. Desde la distancia que podemos observar un evento cualquiera, sin involu- crarnos más que por medio de la observación y con ese simple acto asumimos un lugar y establecemos un adentro y afuera de escena, donde no hay más elementos diferenciadores entre espectador y situación observada, que aquello que permite el encuadre de la mirada. Se señala en La Teatralidad relativo a lo que él desarrolla como la teatralidad cotidiana que ... sería no solamente la aparición de un quiebre en el espacio, de una división de en lo real para que pueda surgir una alteridad, si no la construcción misma de este espacio diseñada por la mirada del espectador (Feral, 6).

Las animitas permiten este encuadre de mirada y poseen además ese carácter de escenario por su propia diferenciación del paisaje, lo que en el caso de las animitas milagrosas no se cierran en la construcción de la animita misma, si no que se amplían, crecen, y su crecimiento es orgánico, dependiendo de las condiciones mismas del espacio adyacente.

Este crecimiento no sólo se da en términos de materia concreta, sino que también es posible identificar otras prácticas o formas de apropiación de tipo vulnerable o perecible, por la precariedad de su condición. Ejemplos de ello son la esperma de velas, los textos y/o pinturas que abarcan un espacio mayor y que sin embargo no limita ni los tránsitos ni cambia la condición de espacio público o privado, sino que simplemente el estar presente lo convierte en ampliaciones espaciales de la animita misma.

Un texto escrito con pintura sobre la calle hace presente tal espacialidad diferenciada. El elemento agregado del texto en la vereda $-\mathrm{y}$ aquí me refiero directamente a la pintura como marca lineal y no al texto por su carga semiótica- que delimita y diferencia un espacio-, hace que el cuerpo lo perciba como parte de la animita misma, sin sobrepasarlo o vejarlo. No es una actitud respetuosa pasar por encima de ese texto aunque no haya un impedimento físico. Ese texto ocupa un espacio sin tener una densidad matérica que justifique la actitud del transeúnte de no pasar por sobre él.

Estas materialidades vulnerables como he decidido llamarlas son una delimitación abstracta que los sujetos rara vez vulneran, ni cuando las personas que hacen uso del espacio no sean practicantes de la religiosidad popular, sin embargo, es la condición matérica o intangible de este tipo de demarcaciones la que está condenada porque su vulnerabilidad corresponde a su propia condición de materia inocua o insubstancial.

\section{EL TIEMPO. LA HISTORIA BORROSA Y SU CONSTANTE REESCRITURA}

...a pocos metros de la Alameda, un asaltante nocturno asesinó a un caballero y el cadáver quedó aquí toda la noche. Debido a la oscuridad, nadie pudo verlo hasta que amaneció.

...venía saliendo del hospital, recuperado de un mal pulmón, cuando lo acuchillaron por robarle una humilde bolsa de utensilios.

...lo único que sé de Rumaldo es que era un joven de veinte años, que lo mataron entre tres hombres.

...era un huasito enfermo que venía saliendo del hospital y llevaba una mantita acuesta; y los malhechores, creyéndole huaso platudo, lo asaltaron y le dieron muerte ${ }^{3}$. 
Claramente ninguna de estas historias coincide. Esto se debe principalmente a que estamos sumamente acostumbrados a pensar en la idea de historia como una gran y única verdad. En el caso de las animitas no sólo nos enfrentamos a las historias polifónicas, sino a que entre todas se construye un gran relato que no tiene nada que ver con las ideas de esa Historia con mayúsculas que aprendimos desde siempre. Lo interesante es que por más que los documentos forenses y policiales den una verdad única del tipo de muerte, no podrán imponerse sobre ésta construcción, es más, si es de conocimiento público esa aparente verdad única del documento, será sumada y no sustitutiva de las ya existentes. Las historias en las animitas son borrosas construcciones de relatos que se suman, no restando validez como podría pensarse, si no que generando una nueva concepción de historia y de verdad, donde las prácticas y las subjetividades plantean la pregunta acerca de qué tan real es creer en una historia objetiva, enfrentada a la que construyen todos los sujetos como una parte viva y consciente del momento y el lugar.

La idea de historia borrosa, en el caso de las animitas se puede leer, en tanto sepamos que quien lee los elementos que componen las animitas, crea una historia por medio de la suma de relatos de otros quienes ya conocen la milagrosidad de tal ánima o la historia de la muerte trágica y su conversión de nexo con lo divino. Con todos estos elementos se complementa la información que éste sujeto ya maneja periféricamente para dar forma a la historia propia, o la que afirmará su construcción y posterior relato. De esta manera, podemos asumir que toda historia es cierta, que las razones por las que se erige el cenotafio es una razón más allá de aquello que exactamente pasó y que su función está entre los practicantes de una religión popular, en relación a la compasión por su calidad de alma en pena o por la necesidad de su carácter limítrofe, para hacer pedidos de favores por medio de ellas a Dios o la Virgen.

Todos estos elementos construyen la relación de espacio y religión, con ellos podemos además atender a la idea que olvidar paulatinamente el hecho trágico y volverlo mítico en la medida que crece la animita, genera una transformación, diferenciando evidentemente el lugar donde ánima y mortales conviven en relación a las capacidades de cada uno. El ánima concede un favor a cambio de reconocimiento, acción o sacrificio de quien ha solicitado sus servicios. Ello se ve materializado de distintas maneras. Una de ellas, por ejemplo y en relación a la "veracidad" de los hechos, tienen que ver con la confirmación de los datos de la historia por medio de las placas de agradecimiento en la animita. En un caso, por ejemplo Romualdito ${ }^{4}$ se encuentran placas de fechas anteriores a la fecha que los mismos practicantes asumen como la de defunción. Esta situación hace pensar que ese sitio haya sido un cruce peligroso, antes de la muerte de este personaje y donde antes ya se habían producido distintos accidentes con resultado de muerte.

\section{UN ESCRITO DE LA LÍNEA Y SU FRONTERA. DIBUJO}

Eso es lo que digo, eso es lo que escribo y es eso solamente lo que se encuentra en las palabras que trazo, $y$ en las líneas que esas palabras dibujan, y en los blancos que deja aparecer el intervalo entre esas líneas... Georges Perec
El dibujo, mi principal lugar de trabajo, satisface mis necesidades de artista. Generalmente se piensa en lo limitado de su formato porque se asume que está acotado a la hoja del papel, sin embargo en mi concepción el dibujo y más aún, la línea, es un constante desplazamiento de esta idea limitante. El dibujo pese a ser una agrupación de elementos sobre la superficie, devela también un constante deseo por salir de ese marco, por ser desplazamientos de sí mismo sobre el paisaje, o de alguna manera, como artista, es donde lo encuentro y lo busco.

Una forma de ver esto sería a partir de una serie de analogías donde, por ejemplo, la animita misma como construcción tangible es una especie de dibujo relacionado más al gesto y la mancha, en la que los límites o bordes están abiertos y quien trae una nueva ofrenda amplía esos bordes o redibuja sobre el mismo dibujo ya hecho, concretamente con la sobreposición de placas, o más sutilmente, el instalar una vela sobre otra que ya casi se apaga. De la misma manera, me atrevería a decir que la historia borrosa de la que hablaba anteriormente es también un dibujo de láminas una sobre otra, donde se sobreimprimen elementos del relato.

Concluiría en pensar que la animita material y su historia borrosa son a lo que podría llamarse, un dibujo inconcluso construido por capas, pero en condiciones de obra abierta que necesita de sus devotos para seguir dibujándose constantemente y borroneando bordes para abrirlos a mayores posibilidades de extensión. Ésta característica de historia borrosa, se parece más a una sobreimpresión que a una línea cronológica, donde la construcción del relato es al mismo los llenos de la memoria y vacíos del olvido (Augé, 27).

La línea como límite o borde, además habla claramente de esas ideas de fronteras permeables a desarrollar, dado que no hay nada más limitante y abierto que una línea. La analogía más básica para esta situación está en el caso de cruzar una frontera política entre dos países, la frontera es una línea que está trazada ficcionalmente en el mapa, pero en el momento que un sujeto desea atravesar esa línea, se consolida como frontera limitante, donde la necesidad de documentos o prohibiciones -tipo de cosas que pueden ingresar o no a un país- se hacen reales.

\section{LAS FRONTERAS PERMEABLES EN LA ANIMITA}

Las animitas en su carácter fronterizo, pueden ser consideradas bajo tres grandes términos, como frontera espiritual, material o de uso. Estas clasificaciones, coinciden con otros nombres en investigaciones como la de Lautaro Ojeda y Miguel Torres donde se les denomina a estas tres grandes nociones como: etapas espirituales, el espacio estructural y el espacio de adherencia. Sin embargo cada una en su categoría presenta una característica particular de permeabilidad, lo que significa que tales fronteras no son estáticas y deben para mantenerse en tal categoría, estar en constante variabilidad. Lo interesante del fenómeno, es que no solamente son variables determinadas, si no que los mismos actuantes pueden hacer de ellas nuevas variantes que reafirman la permeabilidad.

\section{FRONTERA ESPIRITUAL}

La frontera espiritual es una frontera etérea, relativa a la religión. Esta frontera, refiere particularmente a las característi- 
cas del alma para la cual se construye la animita. La condición de alma que sin ritual de extremaunción deambula buscando ese camino hacia la eternidad, es un latente riesgo de convertirse en mal-ánima, perturbando el normal desarrollo de las actividades de quienes viven en las cercanías del lugar. Considérese que en algunos casos no son los familiares quienes levantan la construcción, si no que algún vecino que después de ocurrida la tragedia, se ha visto "penado" con lo que asocia su desventura a la necesidad del alma en pena de encontrar un refugio que la contenga. Con ello, no sólo retoma el control de su propiedad, sino que además consigue la venia del ánima que le estará agradecida, pudiendo concederle favores. De alguna manera ésta es una forma de comunicación amigable entre dos mundos, el de los vivos y el de los muertos, en ese espacio intermedio (donde deambulan las ánimas y que no es ni tierra ni infierno) antes de llegar al Cielo.

Considérese también en las características de este tipo de almas, que su condición fronteriza las dota de poder sobrehumano con el que pueden intervenir por causas mayores, lo que genera entre vivos y ánimas una economía simbólica, donde pedir un favor es a cambio de un reconocimiento, manda o acción compensatoria.

Esta relación se ubica entre vivos y muertos de manera inmaterial pero conviviendo en el espacio y donde además puede llegar a generar cambios experienciales, por medio de la realización del favor, así como del cobro por el no pago convenido hecho el favor.

\section{FRONTERA POR CONVENCIONES DE USOS}

Para hablar de este tipo de frontera en la animita, es necesario aclarar ciertos temas que desde la generalidad de lo que podría ser su enunciación, podría causar confusiones. Hablo principalmente de la idea de espacio público y espacio privado en relación a las consideraciones de lugar y no lugar de Marc Augé desarrolla principalmente en su libro Los no lugares, espacios del anonimato.

Para Augé los no-lugares son espacios exclusivos de la contemporaneidad y que presentan la característica del anonimato entre quienes transitan por ellos, sin temporalidad ni reconocimiento de los sujetos que se mueven en ellos. Las ciudades modernas por su tipo de población, estilo de vida, medios de comunicación, etc. hacen que los espacios de intercambio se hayan ido modificando y en ellos no encontramos más que los espacios que cumplen una función determinada y distanciada de las funciones de antaño, donde el intercambio de persona a persona se hace cada vez más difícil y más distante.

Las consideraciones que establece Marc Augé para determinar qué tipos de espacios responden al concepto de lugar o de un no-lugar son relativas, es decir, no todo en la ciudad contemporánea está delimitado físicamente, hay espacios metafóricos o proyectados que son capaces de albergar, al mismo tiempo, las ideas de espacios públicos o privados dependiendo de la situación particular. Es así como Augé establece una relatividad de relaciones (y no el carácter negativo de uno por el otro) entre aquello que consideramos espacio privado y espacio público y los que él denomina como lugares y no-lugares. Desde este punto de vista cabe señalar entonces, como aquel lugar físico, considerado espacio público totalmente reconocible, puede ser para unos un no-lugar (aque-
Ilos que hacen efectivas las funciones) y para otros un lugar (aquellos que habitan, practican o ejercen funciones permanentes). Es decir, según las propias palabras de Augé el nolugar responde a el espacio creado por la mirada que lo toma por objeto particularmente el mismo sentido de mirada que antes mencionaba en relación a Feral.

Una animita generalmente se encuentra en un espacio público, como una calle, un camino, un roquerío, etc. Estos espacios mantienen sus funcionalidades permanentemente, no varían, sin embargo quienes transitan por tales lugares al enfrentarse a la presencia de una animita, pueden mantener sus actividades mientras que otros pueden variar sus acciones en relación a su presencia. Me refiero a quienes creen en la práctica de las animitas o quienes se sienten identificados con la religión popular, en este sentido su disposición hacia el cenotafio será la de presentar un respeto o saludo, persignarse o evocar alguna frase religiosa.

Obviamente también están quienes tienen como fin de su recorrido el visitar la animita para el pedido de un favor, el pago de una manda o el mantenimiento de la animita en términos de aseo, orden o cambio de agua para las flores, entre otras actividades. De las dos primeras actividades, el pedir un favor o ir a pagar alguno realizado por el ánima, son concretadas por medio de una actitud meditativa, reflexiva, haciendo lugar, lo que podría pensarse que corresponde a una disposición corporal, más del espacio privado por la condición de vulnerabilidad del cuerpo ante el contexto; mientras que otros transitan, usan el espacio sin fines ligados a la religión. La particularidad de esto, es que cohabitan ambas situaciones (lugar y no lugar) así como los tiempos sagrados y profanos en este espacio público con características de lo privado.

\section{FRONTERA MATERIAL Y DE UBICACIÓN}

Dentro de los parámetros de este tipo de frontera, existen dos que se ligan por condiciones externas. La frontera de interior y exterior, dada por el material usado para la construcción de la animita y la frontera entre A y B relacionada al lugar determinado donde se decide ubicar la construcción y que generalmente es un lugar que no moleste al común uso, como por ejemplo entre una reja de jardín y la vereda. Obviamente esta frontera está absolutamente ligada a la idea de frontera por convenciones de uso y determinada por el lugar donde ocurrió la tragedia.

Las animitas son la materialización de un cuerpo perdido, esta característica particular tiene relación con lo ya anteriormente señalado en términos de una muerte violenta o inesperada que implica un corte en el continum del tiempo. La muerte es comprendida bajo los términos de enfermedad o vejez, y aunque existe la conciencia de la mortalidad, lo inesperado ligado a lo violento o injusto reafirma la permanente presencia del factor perecedero en nuestras vidas. Ante esta situación la construcción de las animitas es la creación de otro cuerpo, que presenta características particulares y que es además constantemente actualizada por los familiares o devotos no sólo en términos de cuidado y aseo, sino que también en lo que refiere a la identidad del fallecido en el lugar, así por ejemplo el caso de un niño muerto, no variará esa imagen, por más que pasen los años, y continuará en el imaginario como un niño que necesita estar constantemente actualizado de lo infantil, como en un estado de permanente inocencia. 


\section{INTERIOR-EXTERIOR: PERMEABLE}

Por medio de esta sub-categoría, trataré de desarrollar lo que significa la construcción misma entre la metáfora y una observación de la forma para tal manifestación. Con lo primero me refiero a que la construcción de la casita, gruta u otro le entrega al ánima un lugar donde estar, donde cobijarse, un nuevo cuerpo que habitar, dado que habiendo perdido el propio, busca constantemente uno nuevo. Esta construcción es un lugar propio para el alma, donde sólo caben sus ofrendas y la misma. En esta construcción hay un interior (espacio exclusivo para el alma o privado en términos debatibles) lo que significa que al mismo tiempo hay un exterior. Ambos espacios son usados por el ánima, el primero para recibir petitorios y ofrendas al mismo tiempo que se cobija y ese exterior que usa para ir a cobrar o penar.

Particularmente en la idea de casita, pienso que es una construcción funcional para habitar que genera cierta extrañeza si no se tiene familiaridad con la práctica y que deja abierta la pregunta sin respuesta necesaria, pero curiosa (especialmente para mí como artista) de ser un objeto funcional para algo etéreo, eterno y atemporal.

\section{A. ENTRE A Y B}

Esta clasificación es bastante elemental y tiene que ver con la ubicación de la animita en el territorio. Es de carácter fronterizo o más bien intermedio, ya que se las emplaza en lugares donde no molesten o intervengan el normal funcionamiento del espacio, así se ubican por ejemplo entre una cerca y el camino, en el roquerío, entre calle y vereda.

De estos espacios uno que me llama la atención particularmente, es el de la berma, dado que en sí es un espacio pensado para la eventualidad, para la falla del funcionamiento ideal de una carretera o autopista, la animita en ese espacio es justamente el reflejo de esa falla en el lugar pensado para dicha falla.

Finalmente y con respecto a las fronteras, señalar que cualquier tipo de frontera existe en la medida que haya alguien que quiera cruzarlas, las fronteras permeables, por su parte, juegan constantemente con esa condición, ya que están presentes como fronteras o bordes, pero no impiden el paso de ida ni el de vuelta.

\section{CONCLUSIONES}

Los elementos desarrollados en este artículo son materializados en dos instancias: la experiencia de la animita misma y la Dibujo - Instalación para la muestra colectiva en el Centro de Extensión en Identidad y Patrimonio del Consejo Nacional de la Cultura y las Artes en Valparaíso (noviembre de 2011); muestra que se encontraba en el marco del II Encuentro Nacional Animitas, Identidad y Patrimonio.

Este dibujo - instalación es la evidencia del proceso de este texto, y de la investigación que he realizado durante algún tiempo en espacios de religiosidad ubicados en lugares públi$\cos y$ donde se generan delimitaciones virtuales y diferenciaciones del espacio, en relación a lo público y lo privado. La línea se configura en este trabajo, como un elemento delimitador cultural, como en el ejemplo de la franja trazada en el suelo: de este lado de la línea estamos en un lugar y atravesando la misma ya nos encontramos en otro, sea este otro un país, abordar en metro, etc.

Escogí el dibujo técnico para dar cuenta de un espacio de lleno y vacío que en la animita está muy presente, por la idea de ser una casa para el alma donde claramente la idea de alma es justamente lo incorpóreo del sujeto, por eso delimito, a través del dibujo lineal que es materia inasible, un espacio que apela al inconsciente colectivo. Esas casitas al lado del camino que señalan la presencia de un espacio de religiosidad, con sus particularidades de espacio público y privado. Finalmente para cerrar esta conclusión, agradecer sinceramente la ayuda de quienes montaron este trabajo: Ignacio Quinteros, Álvaro Loins y Felipe Quirós, además de las gestiones y la invitación a participar de Lautaro Ojeda.

FICHA DE TRABAJO

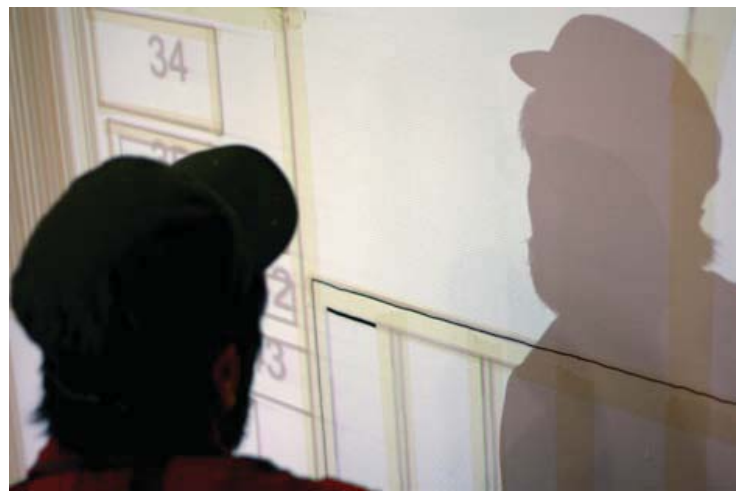

Montaje de obra por Ignacio Quinteros, Álvaro Loins y Felipe Quirós

BORDES DE ÁNIMA.

UN TRABAJO DE LA LÍNEA Y SU FRONTERA

\section{DESCRIPCIÓN}

Dibujo técnico escala 1:1 de una animita real de Valparaíso, construida con plumón negro sobre cinta adhesiva de papel en la pared y suelo. Medidas variables.

El dibujo es una idea de lleno y vacío. Una línea delimita un espacio, las animitas lo diferencian en lo profano. El espacio de los vivos es el del espectador, entendido en la idea de línea como límite, como una separación entre el espacio de lo público y transitable (en este caso la galería) y la virtualización de la animita como un espacio de recogimiento religioso, aunque descontextualizado, vinculado a la idea de instalación y la necesidad del espectador de recorrer la obra e identificar en esa experiencia, una particularidad con respecto a otras acciones cotidianas.

Pienso la animita en sus posibilidades de emplazamiento, así como las razones de su construcción ligadas a muertes trágicas, que se desdibujan del espacio y se construyen como relato en la memoria.

\section{LUGAR DE EXHIBICIÓN}

Centro de Extensión en Identidad y Patrimonio del Consejo Nacional de la Cultura y las Artes en Valparaíso, Chile. Noviembre de 2011. 

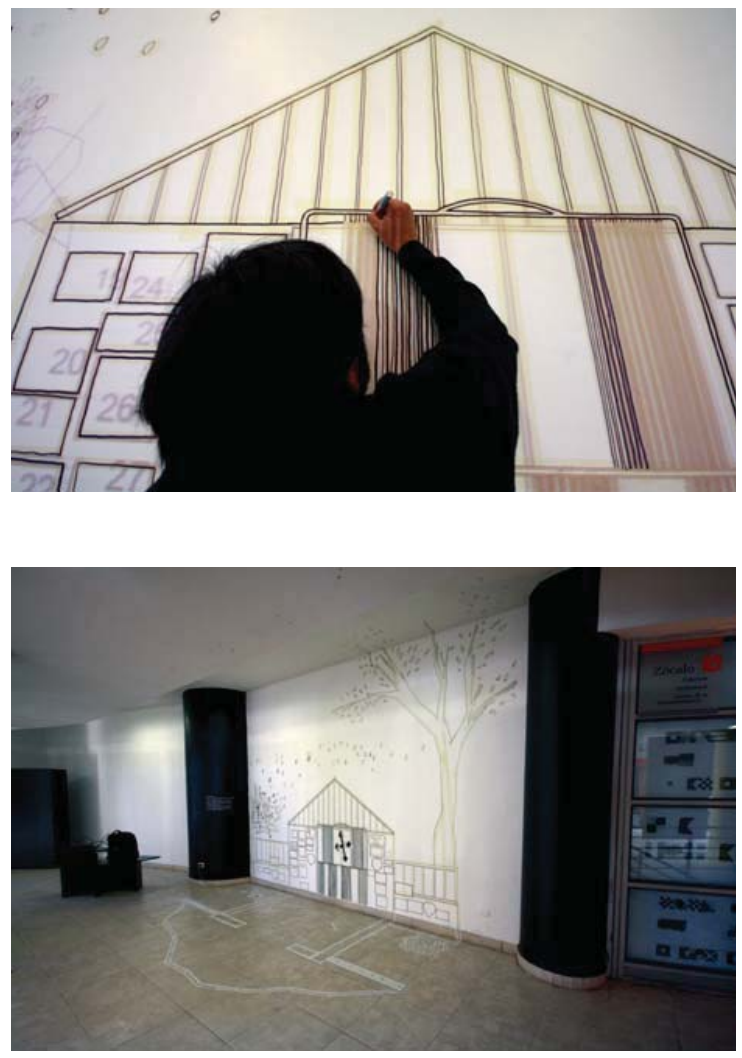

Borde de ánima. Vista general

\section{NOTAS}

1. Los site specific, en palabras simples, corresponden a trabajos de arte que están pensados y funcionan en un lugar determinado, dado que el lugar - como contexto- condiciona la producción.

2. La definición que me interesaría utilizar en este texto, sería la de Boris Groys en su artículo "La topología del arte contemporáneo" en términos de copia y original ligado a la idea aurática de Benjamin y donde señala que El arte de la instalación, que en la actualidad es la forma señera del arte contemporáneo, opera como un reverso de la reproducción. La instalación extrae una copia del presunto espacio abierto y sin marcas de la circulación anónima y lo ubica -aunque sólo sea temporalmente- en un contexto fijo, estable y cerrado de un "aquí y ahora" topológicamente bien definido.

3. Recopilación de versiones sobre la muerte de Romualdito, en L'Animita, Hagiografía Folclórica, Oreste Plath, Editorial Grijalbo 1995.

4. Calle San Borja esquina Alameda. Comuna de Estación Central, Santiago, Chile.

\section{BIBLIOGRAFÍA}

Augé, M. (1996). "Los no lugares, espacios del anonimato una antropología de la sobremodernidad". Barcelona: Gedisa Editorial. (1 a $^{\text {e }}$ dición francesa, NON-LIEUX, introduction à une anthropologie de la surmodernité. Paris, édition du SEUIL, 1992).

Augé, M. (1998). "Las Formas del Olvido". Barcelona: Gedisa.

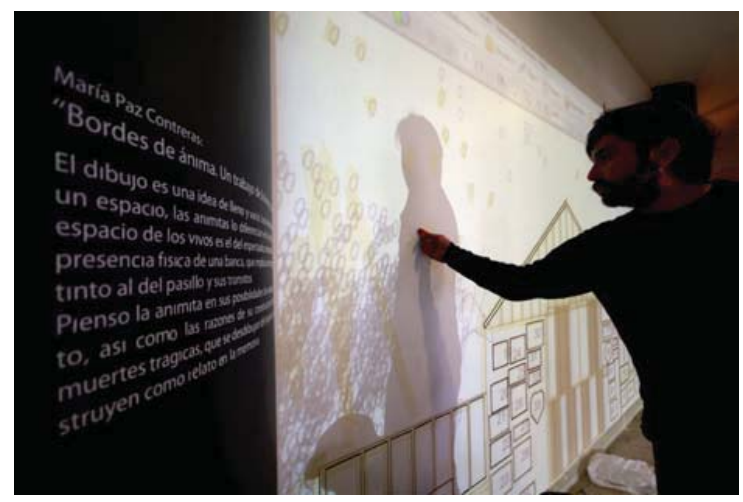

Montaje de obra por Ignacio Quinteros, Álvaro Loins y Felipe Quirós

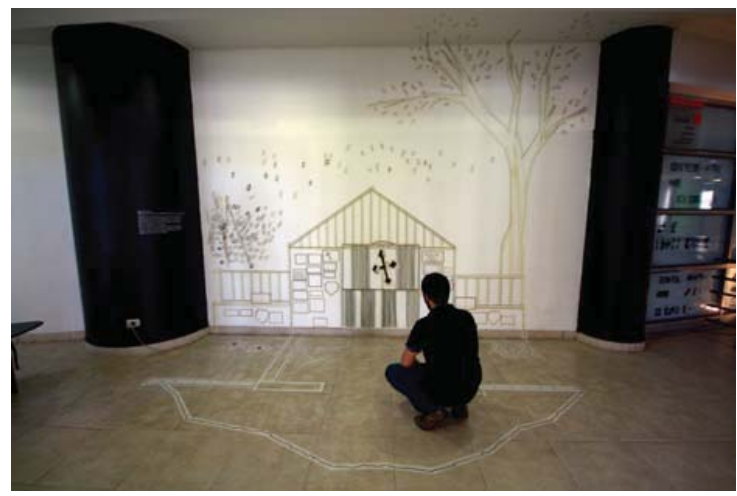

Borde de ánima. Vista forntal

Bourriaud, N. (2006). “Estética Relacional”. Buenos Aires. Adriana Hidalgo Editora.

Feral, J. (2003). "Acerca de La Teatralidad". Buenos Aires: Editoria nueva generación.

Groys, B. (2008) "La topología del arte contemporáneo" en la Antología Antinomies of art an culture. Modernity, posmodernity, contemporaneity. Duke University Press, pp. 71-80.

Lindón, A. (2007). "Los imaginarios urbanos y el constructivismo geográfico: Ios Hologramas espaciales". Revista EURE, 99, pp. 31 46

Plath, 0. (1995). "L'animita: hagiografía folclórica». Santiago: Editorial Grijalbo.

Perec, G. (2007). "Especies de Espacios”. España: Montesinos.

Pérez Riobello, A. (2008). “Merlau-Ponty: Percepción corporalidad y mundo". Revista de filosofía Eikasia, 20, pp. 197-220. recuperado en: http://www.revistadefilosofia.org/

Pires Martins, María Helena. Propuesta de clasificación del gesto en el teatro en Guinsburg, J. (organizador). Semiología do teatro. São Paulo, Editora Perspectiva, 1978.

Merlau-Ponty, Maurice (1945). "Fenomenología de la percepción", Medio digital (6-57) 